\title{
Determinación de la sostenibilidad de la industria mineral de la cerámica y del vidrio mediante herramientas de gestión ambiental
}

\author{
J. A. ESPÍ RODRIGUEZ
}

Univ. Politécnica de Madrid. Madrid, España

\begin{abstract}
Desde hace años se han ido creando una cada vez más numerosa y variada serie de herramientas de gestión ambiental que permiten analizar y calificar ambientalmente cada sustancia mineral y sus condiciones de contorno físico, económico y ambiental. Así, se cuenta con el Análisis de Flujo de Materia (FMA) de una operación minera, el Análisis del Ciclo de Vida (LCA), el Análisis Energético o Exergético, el Análisis Coste Beneficio Ambiental, los Perfiles de Carbono, la Mejor Tecnología Disponible (MTD) y un enorme etcétera que, en el caso de la minería, analizan y clasifican tipologías de yacimiento, disponibilidad de recursos, condiciones de afección ambiental, transparencia en la comunicación y casi todos los aspectos que configuran la geometría ambiental del proceso productivo. El trabajo expone la aplicación de las herramientas de gestión que mejor se acoplan a los minerales de interés en la industria cerámica y del vidrio.
\end{abstract}

Palabras clave: materias primas, procesamiento, propiedades quimicas, gestión ambiental, uso de materia.

Determining the sustainability of ceramic and glass industry using environmental management tools

In the last few years we have seen the creation of an everyday larger and varied series of are creating large and varied series of environmental management tools used to analyze and classify environmentally each mineral and its physical, economic and environmental circumstances. Thus we now, count with the Material Flow Analysis (MFA) of a mining operation, the Life Cycle Analysis (LCA), Energy and Exergy Analysis, Cost Benefit Environmental Analysis, Carbon Footprints, the Best Available Technology (BTA) and many others able to analyze and classify ore deposit site types, availability of resources, environmental impact conditions, transparency in communication and almost all crucial aspects related with the environmental geometry of the mining production process. This paper focuses on the application of some management tools that best fit to the minerals used in the ceramic and glass industry.

Keywords: raw materials, processing, chemical properties, environmental management, material use.

\section{IDEAS GENERALES}

Desde el aspecto ambiental, los minerales industriales se diferencian de las menas metálicas y energéticas en la escasa necesidad de su concentración (eficiencia energética), es decir, una relativa limitación en la masa de sus residuos y en la, generalmente, baja agresividad de sus emisiones $y$, también, en la habitual demanda de su comercialización en seco (gasto energético adicional). Otros factores como el bajo ratio de desmonte en una generalizada minería a cielo abierto, la posible transformación en planta para generar el producto final y otros, configuran el perfil medioambiental de los minerales industriales.

\section{LAS HERRAMIENTAS DE GESTIÓN AMBIENTAL APLICADAS A LOS PROCESOS INDUSTRIALES}

El término de Herramientas de Gestión Ambiental se ha aplicado a un conjunto de procedimientos de variada índole y origen que persiguen el análisis y valoración en términos de sostenibilidad de los procesos industriales, aunque también admite su aplicación a un extenso abanico de actividades humanas.
Desde hace años se han ido confeccionando una cada vez más numerosa y variada serie de herramientas de gestión ambiental que permiten analizar y calificar ambientalmente cada sustancia mineral y sus condiciones de contorno físico, económico y ambiental.

De esta forma, se cuenta con los Indicadores de Sostenibilidad de una operación minera, su Análisis de Flujo de Materia (FMA) aplicado por J.A. Espi y S.A.Moreno (1), el Análisis de Ciclo de Vida (LCA) aplicado por J.A. Espi et al. a los minerales españoles (2, 3 y 4), el Análisis Exergético o Energético, desarrollado por S. A. Moreno a otras especies minerales (3 y 4), el Análisis Coste Beneficio Ambiental, desarrollado y modificado por J. A. Espi y E. Berrezueta (3 y 5), los Perfiles de Carbono, la Mejor Tecnología Disponible (MTD) aplicada a los minerales industriales españoles por Barettino, Obis, Espi y otros (2), la Huella Ecológica, aplicada por J.A.Espí y E. Berrezueta (5), el Perfil de Abatimiento, los Informes Estandarizados de Comunicación y un enorme etcétera que, en el caso de la minería, analizan y clasifican tipologías de yacimiento, la disponibilidad de recursos, condiciones de afección ambiental, transparencia en la comunicación y, 
hoy día, casi todos los aspectos que configuran la geometría ambiental del proceso productivo. Además de alguna de las mencionadas se derivan, ya especializadas, otras que perfilan mejor, aspectos concretos de las actividades productivas. Así, el GER o energía primaria consumida en todas las etapas de ciclo de vida, es una consecuencia del ACV y, también, la actualmente bien difundida, Huella del Carbono.

Estas herramientas constituyen una manera fácil de proceder para clasificar la condición de sostenibilidad de un proyecto de explotación minera.

\section{EL PERFIL DE LA INDUSTRIA DE LOS MINERALES INDUSTRIALES}

Como se ha indicado con anterioridad, en una primera visión desde el aspecto ambiental, los minerales industriales se diferencian de las menas metálicas y energéticas en la escasa necesidad de su concentración (eficiencia energética), es decir, una relativa limitación en la masa de sus residuos, en la, generalmente, baja agresividad de sus emisiones $y$, también, en la habitual demanda de su comercialización en seco (gasto energético adicional). Otros factores de índole general como el bajo ratio de desmonte en una generalizada minería a cielo abierto, la posible transformación en planta para generar el producto final y otros, configuran el perfil medioambiental de los minerales industriales.

\section{LAS HERRAMIENTAS ELEGIDAS}

La realización de los tres estudios que a continuación se presentan, Análisis Coste-Beneficio Ambiental, Análisis de Ciclo de Vida y Análisis de Flujo de Materia y Energía de la extracción y primera transformación de los minerales no metálicos, obedece al deseo de mostrar las posibilidades de tres de las metodologías con las que cuenta el Análisis de Gestión Ambiental, y que de manera muy gráfica explican tanto la aplicación del método como los resultados. A continuación sólo se reflejan los aspectos más importantes de los aspectos elegidos, mostrando tan solo y brevemente alguna etapa de su cálculo.

\section{EL BALANCE COSTE-BENEFICIO AMBIENTAL}

\subsection{Definición}

Una breve definición de este proceso sería el de la construcción de un balance de contabilidad económica, social y ambiental, cuantificado en unidades monetarias; se aborda el estudio separando las partidas del balance en costes y beneficios.

En realidad, el Balance Coste-Beneficio resulta una muy antigua herramienta de análisis que, con la introducción de la consideración ambiental y la introducción en la valoración de aspectos de valores de orden superior a los puramente económicos, ha adquirido una importancia capital en la clasificación sostenible de los procesos humanos de producción.

\subsection{Fase de Inventario}

El trabajo realizado se refiere a la producción de minerales industriales españoles del año 1999, reflejada en la Tabla I.

TABla I. PRODUCCiÓN DE MINERALES IND. EN ESPAÑA

\begin{tabular}{|l|c|}
\hline MINERALES & PRODUCCIONES en t \\
\hline BARITINA & 39.553 \\
\hline CAOLÍN & 326.803 \\
\hline FELDESPATO & 421.700 \\
\hline MAGNESITA CRUDA & 502.209 \\
\hline SALES POTÁSICAS & 915.000 \\
\hline ARCILLAS ESPECIALES & 800.000 \\
\hline TALCO & 117.581 \\
\hline FLUORITA & 136.425 \\
\hline GLAUBERITA & 740.000 \\
\hline THENARDITA & 146.400 \\
\hline CALIZA MICRONIZADA GCC & 414.567 \\
\hline TOTAL & 4.560 .238 \\
\hline
\end{tabular}

Además, ante la variedad de procesos ligados a las características de los minerales extraídos y tratados y las exigencias del mercado, se ha optado por agruparlos en la Figura 1 según las etapas del proceso industrial

\subsection{Los valores considerados}

El procedimiento general contempla todos aquellos factores que influyen en los resultados de una actividad, en este caso la explotación de los minerales ligados a la fabricación de la cerámica y del vidrio y otros más. Todos ellos han de ser valorados en términos homogéneos y por ello se ha elegido una valoración monetaria: euros/año. Esto requiere un esfuerzo de comprensión y de método, pues se reúnen factores directamente mensurables en términos económicos (inversiones, costes de operación, etc.) con variables que necesitan de una suficiente transformación (empleo, seguridad de suministro, siniestralidad, etc.). 
TABLA II. INVERSIONES EN LOS MINERALES INDUSTRIALES

\begin{tabular}{|c|c|c|c|c|c|}
\hline MINERALES & PROD. ANUAL $t$ & \multirow[t]{2}{*}{$\mathrm{INV} / \mathrm{t} €$} & \multirow{2}{*}{$\begin{array}{c}\text { INV. TOTAL } € \\
3.559 .770 \\
\end{array}$} & \multirow{2}{*}{$\begin{array}{c}\text { AMORT. } € \\
323.615 \\
\end{array}$} & \multirow{2}{*}{\begin{tabular}{|c|c}
$\begin{array}{c}\text { INV. REPOSICIÓN } \\
\text { Y MEJORA ( } € \text { ) }\end{array}$ \\
429.788 \\
\end{tabular}} \\
\hline BARITINA & 39.553 & & & & \\
\hline CAOLÍN & 326.803 & 260 & 84.968 .780 & 7.724 .435 & 4.649 .788 \\
\hline FELDESPATO & 421.700 & 101 & 42.591 .700 & 3.871 .973 & 1.326 .391 \\
\hline MAG. CRUDA & 502.209 & 1.150 & 577.540 .350 & 52.503 .668 & 2.027 .054 \\
\hline SALES-POTAS & 915.000 & 235 & 215.025 .000 & 19.547 .727 & 17.552 .083 \\
\hline ARCILLAS-ES. & 800.000 & 200 & 160.000 .000 & 14.545 .455 & 12.074 .600 \\
\hline TALCO & 117.581 & 95 & 11.170 .195 & 1.015 .472 & 1.814 .345 \\
\hline FLUORITA & 136.425 & 130 & 17.735 .250 & 1.612 .295 & 201.998 \\
\hline GLAUBERITA & 740.000 & 120 & 88.800 .000 & 8.072 .727 & 9.917.773 \\
\hline THENARDITA & 146.400 & 120 & 17.568 .000 & 1.597 .091 & 2.647 .745 \\
\hline GCC & 414.567 & 300 & 124.370 .130 & 11.306 .375 & 5.264 .157 \\
\hline TOTAL & 4.560 .238 & 2.801 & 1.343 .329 .175 & 122.120 .834 & 57.905 .722 \\
\hline
\end{tabular}

Inversiones totales / año: $180.026 .556 €$

Todos ellos se analizan respecto a sus efectos de bienestar social, incluyendo claro está, el propio desarrollo económico de la actividad minera.

Puesto que el procedimiento consiste en la construcción de un balance de contabilidad económica, social y ambiental, cuantificado en unidades monetarias, se aborda el estudio separando las partidas del balance en costes y beneficios o entradas, tal como aparece en la Figura 2. En el cuadro que se presenta a continuación aparecen todos los términos considerados, si bien, en algún caso de los intangibles, se ha optado por su valoración solamente en ciertos aspectos.

\subsection{El Análisis: Ejemplos de algunas partidas}

\subsubsection{LOS COSTES}

\subsubsection{Los Costes generados directamente por las extracciones de mineral}

\section{Las Inversiones}

El procedimiento elegido ha sido el de asignar los costes de inversión obtenidos de proyectos nuevos realizados en diversas partes del mundo, a los proyectos en marcha en España, habiéndolos depurado en lo posible y adaptado en cuanto a la escalación de su magnitud. Tal como lo refleja la Tabla II, se le asignó un valor de inversión anual distribuyendo el total de la inversión inicial en 11 años, y dedicando el valor de las inversiones de reposición y mejora según la cantidad reconocida en la estadística minera más reciente (1999).

Otro ejemplo: Los costes de operación y los generales

Para el cálculo de los costes operativos directos se consideran los datos obtenidos de las estadísticas mineras de 1999. Éstos se dividen en costes de personal, materiales y energía. Las carencias de valores, en algún caso, se han suplido con extrapolaciones a la media del grupo

\section{COSTES}

Generados directamente por las propias explotaciones

Inversiones, producidas por la primera instalación industrial o por la renovación de los equipos.

Costes Operativos y Generales, siempre referidos a la operación extractiva, adicionados a los estructurales y los de carácter general.

Ambientales Directos, incorporados a la misma operación minera, tales como los programas de restauración, prevención de impactos y de control ambiental.

\section{Afectando a la comunidad, en general}

Utilización de las Infraestructuras comunitarias: líneas de energía, comunicaciones, etc.

Ambientalmente Inevitables, es decir, aquellos que pueden afectar al medio sin que razonablemente se puedan evitar: impacto paisajístico después de una restauración, pérdida de biodiversidad....

Degradación del valor del terreno por la proximidad de las explotaciones

Otros

Seguridad Personal, positivos o negativos (coste o beneficio), según mejore o empeore la siniestralidad del sector más afín.

Consumos de recursos limitados, tales como la energía eléctrica, combustibles y agua

\section{INGRESOS}

Generados directamente por las propias explotaciones

Venta del producto, extraído y transformado en una primera operación.

Afectando a la comunidad, en general

Transporte (diferencial) hasta los centros de consumo, considerando que las alternativas, excluido el reciclaje, serán soluciones nacionales.

Empleo en la Comunidad, tanto el directo como el inducido generado por esta actividad.

Mejora de los servicios, como viviendas, urbanismo y comunicaciones

Germen de desarrollo local, contando con la fijación de la población, formación laboral, aumento de salarios, etc.

Otros

Control ambiental, siempre que los efectivos disponibles por la comunidad mejoren a aquellos propios de las alternativas consideradas. Calidad de los productos, en el caso de que pudieran demostrarse mejoras considerables respecto a las alternativas de suministro, calidad directamente discutida por el consumidor, seguridad de abastecimiento y reducción de stocks de materias primas.

Figura 2. Componentes del balance. 


\subsubsection{Costes que afectan a la comunidad}

Ejemplo: Utilización de las infraestructuras comunitarias

\section{Infraestructuras eléctricas}

Solamente se considerará aquella que se refiere a la red nacional de transporte eléctrico, haciendo repercutir su coste por kwh consumido.

\begin{tabular}{|l|l|l|}
\hline $\begin{array}{l}\text { Repercusión del gasto de la Red } \\
\text { Eléctrica Nacional, } € / \text { kwh }\end{array}$ & Mwh consumidos & $\begin{array}{l}\text { Coste de } \\
\text { uso en } €\end{array}$ \\
\hline 0,00151 & 281.000 .000 & 425.292 \\
\hline
\end{tabular}

COSTE TOTAL INFRAESTRUCTURAS ELECTRICAS / AÑO: $425.292 €$

\section{Otro ejemplo: Transporte diferencial de productos acabados}

Siguiendo con las simplificaciones, se emplea el siguiente modelo de cálculo:

1. Se eligen los primeros productores de cada sustancia

2. Se fijan los centros principales de consumo o puertos y fronteras

3. Una vez determinadas las distancias recorridas por cada productor elegido, se obtiene el valor de uso de la carretera, multiplicando la distancia media $(501,8 \mathrm{~km})$ por el tonelaje total transportado $(4.560 .000 \mathrm{t})$ y por el valor de mantenimiento y reparación de $1 \mathrm{~km}$ de trazado $(0,001803 €)$, obtenido del cálculo de uso de la red viaria de la CAM y contrastado en la información de la D.G. de Energía y Transportes.

4. Se repite la operación en el supuesto de una importación total de los productos, en este caso calculando las distancias recorridas entre cuatro centros de consumo y cuatro de llegada del exterior, obteniendo la distancia media que recorrería una tonelada importada. Este valor $(455 \mathrm{~km})$, multiplicado por el tonelaje consumido, a partir de la producción nacional $(2.507 .000 \mathrm{t}), \mathrm{y}$ por el coste $/ \mathrm{km}$ de mantenimiento $(0,001803 €)$, producirá el valor de uso vial del producto que no debería ser importado.

5. El valor de uso de transporte diferencial será la diferencia de los anteriores : $2.069 \mathrm{~m} €$

\subsubsection{BENEFICIOS O ENTRADAS}

\subsubsection{Generados directamente por las explotaciones}

\section{Venta de los productos}

El valor de las ventas mostrado en la Tabla III se refiere a los datos estadísticos del anuario del Ministerio de Economía correspondiente al año 1999, sobre productos acabados comercializables.
TABLA III. VALOR DE LOS PRODUCTOS.

\begin{tabular}{|l|c|c|c|}
\hline PRODUCTOS & $\begin{array}{c}\text { UDS. VEN- } \\
\text { DIBLES } \\
\mathbf{t}\end{array}$ & $\begin{array}{c}\text { VENTAS } \\
€\end{array}$ & $€ / \mathbf{t}$ \\
\hline Baritina (Conc.) & 39.553 & 3.559 .770 & 90 \\
\hline Caolín (P. Lavado) & 326.803 & 22.245 .231 & 68,1 \\
\hline Feldespato (Conc.) & 421.700 & 12.801 .558 & 93,8 \\
\hline Magnesita (Cruda) & 502.209 & 60.265 .080 & 120,0 \\
\hline Sales potásicas (Cloruro) & 915.000 & 109.800 .000 & 120,0 \\
\hline Arcillas especiales.(Preparada) & 800.000 & 78.131 .574 & 97,7 \\
\hline Talco (Preparado) & 117.581 & 11.590 .518 & 98,6 \\
\hline Fluorita (Conc. Ácido) & 136.425 & 12.801 .558 & 93,8 \\
\hline Glauberita (Precipitado) & 740.000 & 64.510 .746 & 87,2 \\
\hline Thenardita (Precipitado) & 4.560 .238 & 438.630 .087 & 96,2 \\
\hline GCC (Prep. ultrafino) & & 13.176 .000 & 90,0 \\
\hline TOTAL & & $49.748 . .052$ & 120,0 \\
\hline
\end{tabular}

VENTA DE MINERALES / AÑO: 438.630.087 €

\section{Calidad de los productos}

Pueden considerarse factores de beneficio tales como las mejoras respecto a las alternativas de suministro, la calidad directamente discutida por el cliente, la seguridad de abastecimiento y la reducción de stocks de materias primas. De entre ellas, se elige una directamente cuantificable, el beneficio producido por la reducción de stoks de materias primas minerales, en la industria española de transformación. El modo de operar ha sido el siguiente: cálculo del ahorro producido por reducción de materias primas en almacén, desde 4 meses a 1,5 meses. Para ello, se parte del valor de la producción nacional y se determina el valor de la compra de 2,5 meses de productos minerales. A este capital se le remunera con un $7 \%$ en los 2,5 meses ahorrados.

\begin{tabular}{|c|c|c|}
\hline $\begin{array}{c}\text { Valor de un mes de la } \\
\text { producción nacional } \\
\text { en } €\end{array}$ & $\begin{array}{c}\text { Tipo de } \\
\text { interés } \\
\% \text { anual }\end{array}$ & $\begin{array}{c}\text { Remuneración en 2,5 } \\
\text { meses } \\
\text { en } €\end{array}$ \\
\hline 36.552 .500 & 7 & 533.000 \\
\hline
\end{tabular}




\subsection{El Balance}

La Tabla IV resume los resultados de los cálculos y detalles no ofrecidos aquí por falta de espacio.

TABLA IV. BALANCE TOTAL.

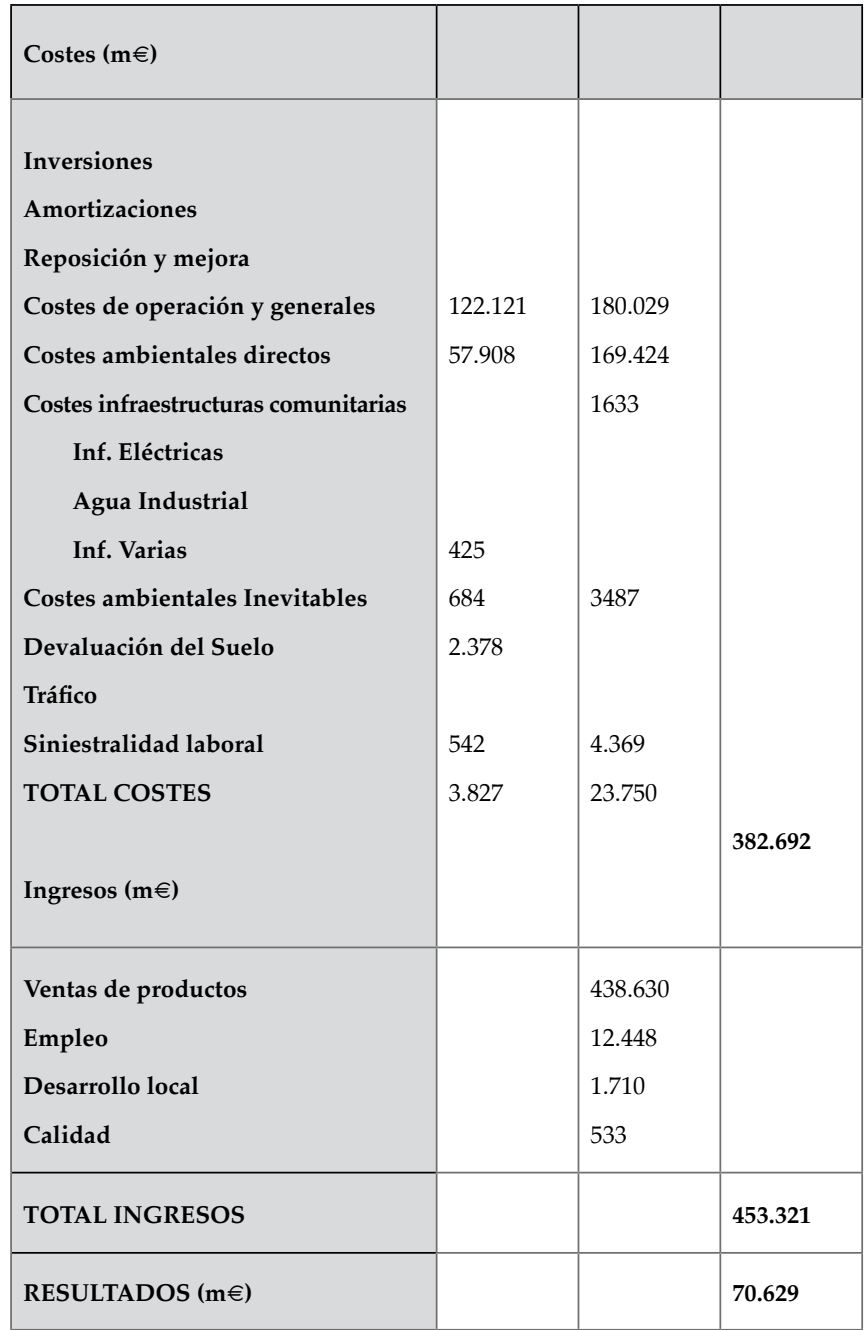

\subsection{Conclusiones}

- Los resultados finales del balance se muestran bastante elevados, mas de un $15 \%$ sobre el valor de las ventas y alrededor de un $40 \%$ sobre las inversiones. Esto supera con creces lo que actualmente es normal en otros sectores de la industria minera.

- La consideración de los factores sociales y ambientales apenas hace cambiar el aspecto anterior. Es más, si no se considerasen los costes de las bajas por enfermedades laborales y accidentes en el trabajo, comunes a toda actividad productiva, los beneficios sociales y de desarrollo superan al cálculo de los costes ambientales.

- Los costes ambientales más llamativos son los llamados "inevitables" que constituyen el 13,5\% del margen económico generado. Los mayores beneficios considerados son los derivados del empleo, que alcanzan el 17,6 \% de dicho margen. Ciertamente, el valor del empleo inducido resulta determinante en esta cifra, y convendría asegurarse en el índice aplicado.

\section{EL ANÁLISIS DEL CICLO DE VIDA DE LAS MATERIAS PRIMAS MINERALES NO METÁLICAS EN ESPAÑA}

\subsection{Definiciones y Conceptos}

El Ciclo de Vida de las Materias Primas es una cuenta, agregada y clasificada, de los bienes y servicios que comprende la fabricación industrial de un bien (en este caso una materia prima mineral), desde que se extrae en la mina hasta que, trasformado o incorporado varias veces a otros sistemas, se abandona definitivamente.

Durante el flujo o vida industrial, los procesos que le afectan consumen otros materiales, energía y dedicación humana (este último insumo es necesario considerarlo si se pretende analizar el impacto social en su integridad), pero además, el desarrollo de la fabricación va causando impactos sobre los entornos naturales con los que se relaciona, además de producir residuos, reciclables o no.

El ámbito del análisis no se circunscribe exclusivamente al entorno directo de la fabricación, sino que también incluye los efectos provocados por la elaboración de otros componentes que se utilizan en la fabricación del producto considerado, es decir, que inicia una cadena de afecciones al medio, cuyo comienzo es la extracción de los materiales básicos de la fabricación, incluyendo los componentes energéticos. Para ello es necesario contar con potentes bases de datos que faciliten el cálculo de las cadenas de procesos de cada componente.

La calidad de las bases de datos en donde se apoya el análisis resulta totalmente decisiva. Afortunadamente cada vez se dispone de más información sobre emisiones y sus condicionantes ambientales. La elaboración de un buen análisis requiere también de un enconado esfuerzo de un grupo de especialistas y de un dilatado tiempo de ejecución. La determinación de alternativas óptimas, y la elaboración de estudios de eficacia y análisis comparativos necesita de programas informáticos específicos, a veces de dificultosa manipulación

\subsection{Bases para el estudio del ACV de los minerales industriales en España}

De la misma manera que en el ACBA, se ha considerado, de la producción minera española, aquellas sustancias minerales que sin restricción alguna pueden encuadrarse en esta categoría, todas ellas con producciones referidas al año 1999 y mostradas anteriormente ya que las aplicaciones comenzaron a realizarse a partir del año 2002 con los datos disponibles de la estadística minera oficial de España, quedando ahora pendiente su actualización, siempre variable según las importantes variaciones de la coyuntura económica actual.

La clasificación de sustancias significa un esfuerzo para agrupar a tan diferentes minerales $\mathrm{y}$, para ello, se tiene en cuenta la realizada en el ACBA sobre los minerales no metálicos españoles. 
7.3 Desarrollo de un Análisis del Ciclo de Vida de extracción y preparación de los minerales industriales de España

\subsubsection{CICLO GENERAL}

En la Figura 3 se representan las tres etapas fundamentales en que pueden dividirse las operaciones de la industria de los minerales no metálicos. De las tres, sólo se desarrollará la primera, la que comprende las etapas de extracción y beneficio, sin que con ello se pretenda disminuir la importancia de contar con el estudio del ciclo completo, si bien la reincorporación de estos materiales, una vez utilizados, a otro sistema productivo, resulta realmente raro, siendo más normal ver como acaba su ciclo en un vertedero de productos inertes.

\subsubsection{ANÁLISIS DE LAS ETAPAS DEL PROCESO}

De igual manera que anteriormente se ha presentado, con la intención de ejemplarizar, se adjuntan alguna de las muchas etapas de cálculo de los procesos que conducen a la valoración final.

En las primeras etapas de cálculo se determinan las emisiones de los insumos gastados en el proceso de la etapa a analizar. Por ejemplo, las emisiones de un litro de gasoil consumido en el acarreo del mineral en cantera.

Después, a cada proceso de la correspondiente etapa, se contabilizan las emisiones provocadas por el consumo de todo tipo

\subsubsection{EL BALANCE FINAL}

El resultado buscado se refiere a la cuenta de emisiones y consumos de sustancias que afectan de alguna manera al medio ambiente, contabilizando cada una de las cuentas parciales. Además, en una primera visión, resulta del todo de interés mostrar, de forma comparativa, los consumos de cada modalidad de explotación. De esta manera, se puede destacar la diferenciación de la minería por disolución, caracterizada

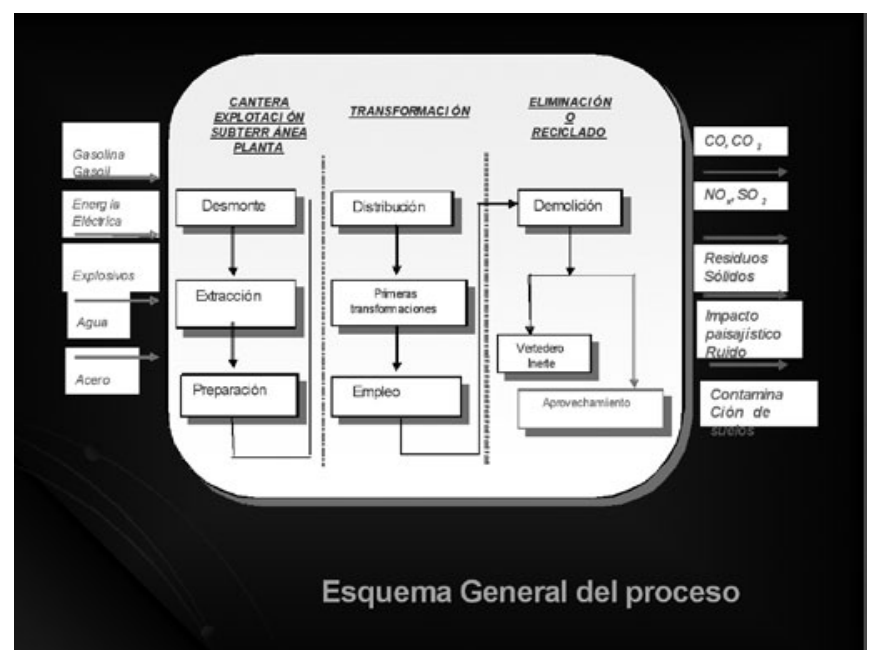

Figura 3. Esquema General de las etapas que conforma el Ciclo de Vida de los procesos de extracción y preparación de los minerales industriales

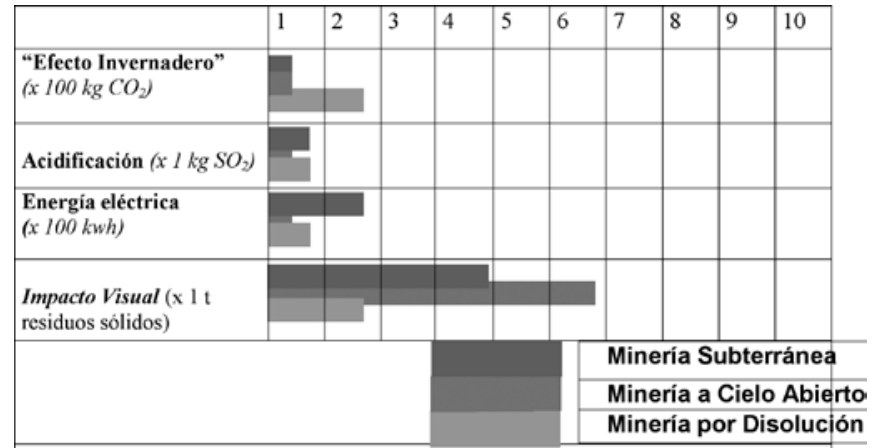

Figura. 4. Efectos totales en las tres alternativas de minería, reducidos a conceptos fácilmente comprensibles.

por un muy elevado consumo eléctrico y también es digno de señalar las notables cantidades de combustibles líquidos en las dos minerías restantes, si bien, en el caso del cielo abierto, una parte se justifique, ambientalmente hablando, por la cogeneración eléctrica. Estos consumos tendrán una implicación en las emisiones de productos agresivos al medio ambiente, como se expone a continuación.

Con el fin de hacer más didácticos los resultados, y además, agrupar los impactos causados por las emisiones, en la Figura 4 se presentan los efectos fundamentales que son consecuencia de las emisiones calculadas. Así, se descubre el bajo consumo relativo de la energía eléctrica en la minería de cantera, el importante "efecto invernadero" provocado por la combustión del fuel oil en la minería por disolución y el importante (y conocido) efecto visual de las minerías tradicionales. Esta contabilidad también puede ser sustituida por una valoración numérica dando "pesos" a cada uno de los factores y sumándolos después.

\section{EL USO DE MATERIA Y EL METABOLISMO INDUSTRIAL}

El metabolismo es un concepto biológico que se refiere a los procesos internos de un organismo vivo. Los organismos mantienen un intercambio continuo de materias y energía con su medio ambiente que permiten su funcionamiento, crecimiento y reproducción. De manera análoga, los sistemas sociales convierten las materias primas en productos manufacturados, en servicios y, finalmente, en desechos.

Según la socióloga y economista austriaca Marina Fischer-Kowalski (6 y 7), el análisis del metabolismo de la sociedad proporciona un marco para distinguir entre culturas, sociedades o regiones, según sus relaciones características de intercambio con la naturaleza. El metabolismo de la industria es un conjunto de procesos físicos integrados que convierten las materias primas y la energía, con la mano de obra, en productos acabados y residuos, en condiciones más o menos estables. El lado de la producción, por si misma, no se autoregula. La estabilización del sistema se consigue mediante la acción humana. Su papel posee dos aspectos: directo, como mano de obra, e indirecto, como consumidor. 
Según Marina Fisher (6 y 7) “El sistema alcanza la estabilidad cuando suministro y demanda se compensan, a través del mecanismo de los precios. Así, el sistema económico es, en esencia, el mecanismo de la regulación metabólica".

El perfil metabólico característico "puede ser utilizado para comparar diferentes modos de producción" (cazadores y recolectores, sociedades agrícolas) en una amplia perspectiva histórica. También puede servir como una evaluación "rápida " de la presión que una sociedad ejerce sobre el medioambiente. Esto contribuye a nuestra comprensión de las interrelaciones de los procesos naturales, sociales y económicos que son relevantes para el desarrollo sostenible. De esta manera, la productividad material total de un sistema socioeconómico es una medida razonable de la presión que ejerce sobre el medio ambiente.

\section{EL "USO DE LA MATERIA" (MFA) Y "LA ENERGÍA" (GER) EN LA EXTRACCIÓN Y TRATAMIENTO DE LOS MINERALES INDUSTRIALES ESPAÑOLES}

\subsection{Los conceptos}

La variedad de circunstancias que concurren en la explotación de los minerales no metálicos es enorme, pese a lo cual la aplicación del análisis de consumo de materia y energía contribuye a caracterizar la posición ambiental de este sector y ayuda a dibujar el perfil energético y másico de la industria. Una guía de comportamiento en los próximos años es la idea de la desmaterialización.

Los materiales empleados por las economías poseen diversos pesos cuando los agregamos en nuestros cálculos. Algunos de ellos, con bajo impacto ambiental, pueden sustituir a los de alto, incluso aumentando la cantidad global, y aun así, se estará produciendo un proceso de desmaterialización. Para construir un buen sistema de indicadores que definan este concepto resulta esencial contemplar las relaciones entre flujos de materia y sus stocks. Resulta un concepto que puede ser contemplado desde varias visiones: proceso industrial, una firma, sector, un país, el mundo entero. Estos conceptos han sido abordados bajo el patrocinio de la Unión Europea y existen muchos autores abordando aspectos muy variados de estos indicadores de sostenibilidad (8 y 9)

\subsection{Los indicadores del consumo de materia}

Para medir la cantidad de materia que una sociedad consume se ha construido una serie de indicadores básicos:

El TMR (Total Material Requirement) consiste en la masa total de las materias primas extraídas de la naturaleza para sostener las actividades humanas. Por ello, TMR se usa como un buen indicador de las bases materiales de una economía.

TABla V. MOVIMIENTOS DE MASA EN LAS EXPLOTACIONES DE MINERALES INDUSTRIALES ESPAÑOLAS.

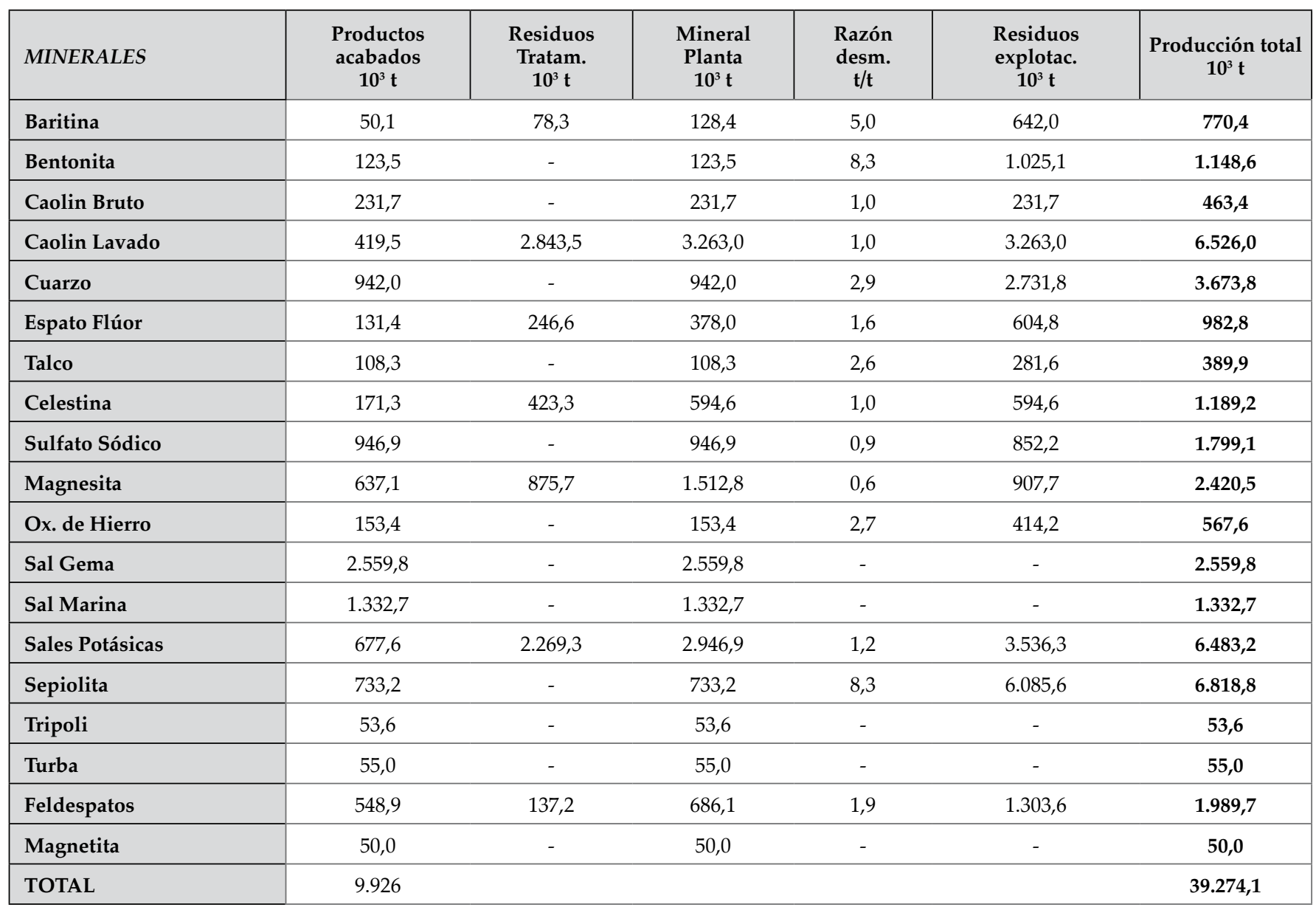


El valor del TMR indica la presión genérica en el medio ambiente, suministrando información similar a la obtenida en el cálculo de las necesidades de agua o de energía

En 1995 el TMR europeo alcanzaba 18,1 miles de millones de toneladas o 49 toneladas por habitante. Debido a su estructura de consumo de materias y a sus "hidden flows" (flujo de materia no evidente, como las toneladas de desmonte asociadas a una tonelada de carbón), los TMR europeos están dominados por la energía, los metales y las sustancias minerales. La razón más importante de presentar Europa (49t/ habitante) un TMR inferior al de USA (84t/habitante) se debe a un menor uso del carbón que en Norteamérica.

\subsection{Aplicación a los minerales industriales españoles}

La cantidad de productos acabados procedentes de la explotación de los minerales no metálicos no es excesiva, no llega a los diez millones de toneladas por año, a pesar de que España puede ser considerada un gran productor de minerales industriales. Sin embargo, en el balance de materia movida por esta industria habrá que considerar la llamada materia oculta y asociada ("rucksack") que en nuestro caso procederá de los siguientes conceptos:

1. Desmontes de escombros asociados al desmonte general en la minería a cielo abierto y muy moderadamente a la minería subterránea.

2. A los residuos de la concentración cuando el proceso y el mineral lo requiera

3. Al incremento de la erosión por limpieza de la cubierta vegetal asociada al sistema productivo

Al ser consideras las toneladas ocultas, tal como aparecen en la Tabla V, el valor de movimiento total cambia sustancialmente:

Las 39,27 millones de toneladas movidas por la minería no metálica surgen de aplicar una recuperación usual a los materiales de donde proceden los productos acabados y de considerar el desmonte necesario para obtener el mineral que entra en la planta de tratamiento. A lo largo de ese proceso se generan residuos procedentes de la concentración mineral en la planta de proceso y los escombros de exterior o de explotación subterránea, aplicando la razón de desmonte obtenida de las estadísticas oficiales.

Los indicadores de uso de materia no producen identificaciones de grupos de manera muy clara. Veamos, por lo tanto, si el consumo energético puede resolver la cuestión. Para ello se compara la producción final de los materiales vendibles con el consumo específico de energía, que incluye tanto los kwh consumidos en la producción de una tonelada de material vendible como la conversión equivalente en kwh de los combustibles gastados en el proceso. La comparación aparece en la Tabla VI.
TABla VI. CONSUMO ENERGÉTICO DE LA PRODUCCIÓN ESPAÑOlA DE MINERALES INDUSTRIALES.

\begin{tabular}{|c|c|c|}
\hline mINERALES & $\begin{array}{l}\text { Productos acabados } \\
\qquad 10^{3} \mathrm{t}\end{array}$ & $\begin{array}{l}\text { Consumo energético } \\
\text { específico Kwh/t }\end{array}$ \\
\hline Baritina & 50,1 & 23,7 \\
\hline Bentonita & 123,5 & 179,5 \\
\hline Caolin Bruto & 231,7 & \\
\hline Caolin Lavado & 419,5 & 39,5 \\
\hline Cuarzo & 942,0 & 43,6 \\
\hline Espato Flúor & 131,4 & 35,9 \\
\hline Talco & 108,3 & 62,0 \\
\hline Celestina & 171,3 & 65,8 \\
\hline Sulfato Sódico & 946,9 & 294,3 \\
\hline Magnesita & 637,1 & 10,4 \\
\hline Ox. de Hierro & 153,4 & 28,4 \\
\hline Sal Gema & $2.559,8$ & 16,5 \\
\hline Sal Marina & $1.332,7$ & \\
\hline Sales Potásicas & 677,6 & 61,9 \\
\hline Sepiolita & 733,2 & 179,5 \\
\hline Tripoli & 53,6 & 85,5 \\
\hline Turba & 55,0 & \\
\hline Feldespatos & 548,9 & 102,7 \\
\hline Magnetita & 50,0 & \\
\hline TOTAL & 9.926 & \\
\hline
\end{tabular}

Caracterización de grupos

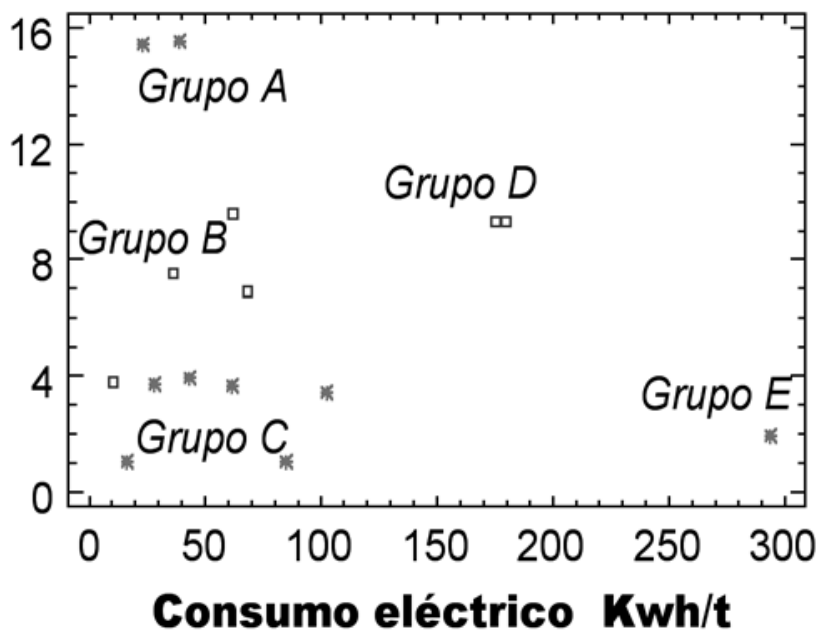

Figura 5. Agrupación de los minerales industriales según su consumo másico y energético 
Al representar en la Figura 5 ambos parámetros de sostenibilidad del proceso en un solo diagrama, se obtiene las siguientes agrupaciones:

Y trasladándolo a una matriz explicativa que figura como Tabla VII,

TABLA VII. EXPLICACIÓN DE LAS AGRUPACIONES OBTENIDAS.

\begin{tabular}{|l|l|l|}
\hline & \multicolumn{1}{|c|}{ Minerales Industriales } & \multicolumn{1}{|c|}{ Características } \\
\hline Grupo A & Baritina y Caolín Lavado & $\begin{array}{l}\text { En su proceso se utilizan métodos no consumidores de energía, pero } \\
\text { sin embargo, se desmonta gran cantidad de materia inservible, ade- } \\
\text { más de un importante proceso de concentración mineral }\end{array}$ \\
\hline Grupo B & Magnesitas, Espato Flúor, Celestina y Sales Potásicas & $\begin{array}{l}\text { Todos sufren un proceso de molienda y flotación, gran consumido- } \\
\text { res de energía, pero sin embargo el desmonte producido resulta no } \\
\text { excesivo }\end{array}$ \\
\hline Grupo C & Óxidos de Hierro, Cuarzo, Talco y Feldespatos Potásicos & $\begin{array}{l}\text { Moderado desmonte durante la explotación en mina y energía va- } \\
\text { riable, dependiendo del sistema de concentración }\end{array}$ \\
\hline Grupo D & Sepiolita y Bentonita & $\begin{array}{l}\text { Elevado desmonte en cantera y considerable consumo de energía } \\
\text { por secado y manipulado en la misma planta de tratamiento }\end{array}$ \\
\hline Grupo E & Sulfato Sódico & $\begin{array}{l}\text { Elevado coste energético debido a la disolución en caliente y secado } \\
\text { final del producto }\end{array}$ \\
\hline
\end{tabular}

\subsection{Consideraciones a partir del análisis de materia y energía}

Los minerales industriales, desde el punto de vista del gasto de materia, señalan su ventaja sobre otros recursos naturales no renovables, debido a sus bajos Índices de enriquecimiento (tratamiento).

La industria se muestra incapaz de apreciar aquellos grandes consumidores de energía, y más aún, de los importantes consumidores de materia. El suministrador actúa a la vez sobre el control de sus costes y sobre la calidad del producto, tratando así de ganar una diferenciación de sus competidores.

El Perfil Energético y de Consumo de Materia de una empresa o sector resulta fácilmente cuantificable y resulta factible la elección de estrategias de elección de calidades de yacimientos en cuanto a consumo materia se refiere. Además, las políticas de búsqueda de tecnologías de bajo consumo de materia y energía coinciden con los objetivos de alcanzar mejores resultados económicos y sociales.

En la actualidad existe una gran variedad de autores que abordan a escala nacional la problemática derivada del uso de la materia, tal como de manera global desarrolla en Europa el grupo GUA, GmbH de Viena (9). Además y con carácter más general también sobresalen trabajos de importancia como los de S. Bringezu y H. Schütz (10), sobre Análisis de Flujo de Materia y la reciente tesis doctoral de Alicia Valero (11) en el entorno de la evolución exergética del mundo mineral.

\section{ALGUNAS CONCLUSIONES SOBRE LA OPORTUNI- DAD DE UNA PRODUCCIÓN NACIONAL}

La consideración de la producción nacional como alternativa de interés para la sociedad española se desprende de la aplicación de las Herramientas de Gestión Ambiental. Recordando algunos conceptos y añadiendo otros, se concluye que:

- La promoción de una producción de las materias primas minerales nacionales se justifica con claridad atendiendo a los siguientes conceptos:

- El carácter estratégico de algunas de ellas, es decir, la dependencia del suministro propio a causa de su estricta adecuación a las peticiones del mercado o bien, la elevación de su precio en los mercados internacionales debido al precio del transporte o a las limitaciones de la oferta.

- La rápida adecuación del producto a las necesidades del consumidor, factor trascendente en la industria de los minerales no metálicos. Además, la facilidad de comunicación con el productor que redunda en una atenuación de los riesgos de variaciones de los precios o de corte de suministros de las calidades demandadas.

- La reducción de los stocks de carácter estratégico para compensar en muchos casos el riesgo de abastecimiento.

- La corresponsabilidad en las medidas de protección ante el riesgo personal en el trabajo y la sensibilidad ambiental del entorno minero, que, por supuesto, no siempre aparecen en otros lugares del mundo. 


\section{BIBLIOGRAFÍA}

1. J.A. Espi y S.A. Moreno. “El uso de la materia y la energía en la extracción y tratamiento de los minerales industriales españoles" Tecnoambiente. 159, 34-40 (2006)

2. D. Barettino, J. Obis y J.A. Espi. Editores. “La industria extractiva no energética y el medio ambiente en el marco del desarrollo sostenible" Instituto Geológico y Minero de España. Madrid. 335 pág. (2005)

3. S.A. Moreno y J.A. Espi. Editores. "Introducción al uso de las herramientas de gestión ambiental aplicadas a los recursos naturales no renovables". 229 pág. Red Desir y OEI. Madrid (2008)

4. J.A, Espi y E. Seijas “El Análisis del Ciclo de Vida aplicado a los materiales de construcción: El granito ornamental de la Comunidad de Madrid". Roc Máquina. Feb.2003. 22-28 (2003)

5. J.A.Espí y E.Berrezueta "El análisis de la gestión de los recursos naturales: La Huella Ecológica como medida del esfuerzo de la naturaleza" en Agua, Minería y Medio Ambiente. Libro Homenaje al Pr. Rafael Fernández Rubio. Instituto Geológico y Minero de España. pp. 685-706. (2005)
6. M.Fischer-Kowalski.. "Society's Metabolism. The Intellectual History of Material Flow Analysis, Part I, 1860 - 1970", Journal of Industrial Ecology, 2, No. 1, 61-78. (1998)

7. M.Fischer-Kowalski, C. Amann. "Beyond IPAT and Kuznets Curves: Globalization as a Vital Factor in Analysing the Environmental Impact of Socio-Economic Metabolism", in: Population and Environment, 23: 7-47. (2001).

8. G. Finnveden and A.Moberg. "Environmental Accounts and Material Flow Analysis and other Environmental Systems Analysis Tools". FMS, Swedish Defence Researh Agency (FOI) and Stockholm University. (2001)

9. GUA (2000) "Analysis of the Fundamental Concepts of Resource Management". www.europa.eu.int/comm/environment/enveco/waste/guareport

10. 10. Bringezu, S., Schütz, H. "Total Material Requirement of the European Union.

11. European Environment Agency". Copenhagen. (2001).

12. 11. A.Valero "Exergy Evolution of the Mineral Capital on Earth". Tesis Doctoral. (2008).

Recibido: 20/12/2010

Aceptado: 26/5/2011 